\title{
Tuberculosis sistémica con insuficiencia suprarrenal: Una presentación olvidada de una enfermedad frecuente
}

\author{
Systemic tuberculosis with adrenal insufficiency: A forgotten presentation of a common disease
}

\author{
César R. Carpio-Chanamé', Miguel Villegas- \\ Chiroque ${ }^{2}$, Halbert C. Sánchez-Carrillo ${ }^{3}$
}

Carpio-Chanamé CR,Villegas-Chiroque M, Sánchez-Carrillo HC. Tuberculosis sistémica con insuficiencia suprarrenal: Una presentación olvidada de una enfermedad frecuente. Rev Soc Peru Med Interna. 2021;34(4): 173-176. DOI: https://doi.org/10.36393/spmi.v34i4.633

\section{RESUMEN}

Varón de 47 años, con híperpigmentación de piel y mucosa oral, astenia, náuseas, vómitos, pérdida ponderal, dolor abdominopélvico e hipotensión. Además, orquiepididimitis y fístula escrotal. En sangre: hiponatremia, cortisol plasmático disminuido y ACTH elevado. Radiografía torácica normal, baciloscopía negativa. La tomografía reveló incremento de dimensiones en la cápsula suprarrenal derecha y pequeños nódulos adrenales en la izquierda. Fue sometido a orquiectomía y la histopatología confirmó tuberculosis urogenital. La insuficiencia suprarrenal fue considerada como parte de una tuberculosis diseminada. La respuesta a la terapia antituberculosa fue exitosa. Este caso es una presentación inusual de una enfermedad frecuente.

Palabras clave: tuberculosis, insuficiencia suprarrenal, enfermedad de Addison, urogenital, orquiepididimitis

\section{ABSTRACT}

A 47-year-old male with hyperpigmentation of the skin and oral mucosa, asthenia, nausea, vomiting, weight loss, abdominopelvic pain and hypotension. In addition, orchiepididymitis and scrotal fistula. In blood: hyponatremia, decreased plasma cortisol and elevated ACTH. Normal chest $X$-ray, negative smear.The tomography revealed increased dimensions in the right adrenal capsule and small adrenal nodules in the left. He underwent an orchiectomy and histopathology confirmed urogenital tuberculosis. The adrenal insufficiency was considered to be part of disseminated tuberculosis. The response to TB therapy was successful. This case is an unusual presentation of a common disease.

Keywords: tuberculosis, adrenal insufficiency, Addison's disease, urogenital, orchiepididymitis

Médico internista. Departamento de Emergencia y Áreas Críticas, Servicio de Medicina Interna, Hospital Regional Lambayeque, Chiclayo, Lambayeque, Perú.

2 Médico infectólogo. Servicio de Enfermedades Infecciosas, Hospital Regional Lambayeque. Investigador, Universidad Nacional Pedro Ruiz Gallo, Chiclayo, Lambayeque, Perú.

3 Médico residente. Servicio de Medicina Interna, Hospital Regional Lambayeque, Chiclayo, Lambayeque, Perú.

\section{INTRODUCCIÓN}

La tuberculosis suprarrenal es de rara presentación y más aún en el contexto de tuberculosis sistémica. Ocurre solo en $6 \%$ de los pacientes con tuberculosis activa. Se sabe que sigue siendo de difícil diagnóstico, es infradiagnosticada y muchas veces solo son halladas en estudios post 
mortem. Se desconoce cabalmente el tropismo del bacilo por las glándulas suprarrenales, pero se refiere que logra subsistir latente durante décadas y en el momento de las manifestaciones clínicas puede haber destrucción hasta del $90 \%$ de las mismas ${ }^{1,2}$

La tuberculosis sistémica que cursa con compromiso urogenital, en la mayoría de los casos, simula clínicamente otras lesiones testiculares, como un tumor testicular, un infarto testicular o, incluso, una torsión testicular. Los individuos de mediana edad, son los más afectados y se presentan con inflamación escrotal dolorosa o indolora. La mayor prevalencia es en el epidídimo, seguido por las vesículas seminales, próstata, testículo y conducto deferente, en ese orden de frecuencia. ${ }^{3}$ La terapia antituberculosa comprende rifampicina, isoniazida, pirazinamida $\mathrm{y}$ etambutol como pilares del tratamiento. ${ }^{4}$

A continuación, presentamos el caso inusual de un paciente con tuberculosis sistémica y con insuficiencia suprarrenal.

\section{PRESENTACIÓN DEL CASO}

Varón de 47 años, natural de Monsefú, Lambayeque, al norte del Perú, enviado a nuestro departamento de medicina interna con historia de dos años de cambios en la pigmentación de la piel, además de disminución de peso de $20 \mathrm{Kg}$ durante los últimos seis meses, asociado a malestar general, anorexia y náuseas. Tres meses antes, experimentó un dolor sordo a nivel lumbar, abdominopélvico y perineal que se intensificó con el paso de los días. Posteriormente, se agregó disuria y hematuria. Acudió a un empírico quien brindó medicación no referida y sus molestias cedieron parcialmente. Los síntomas persistieron y un mes antes de su atención desarrolló una lesión pustulosa a nivel del escroto izquierdo, asociado a dolor que se intensificó, con aumento de volumen y drenaje de material purulento. El paciente no tenía antecedentes médicos notables, alergias conocidas o epidemiológicos importantes.

A la exploración física, peso de $46 \mathrm{~kg}$ y talla $152 \mathrm{~cm}$. Presión arterial 90/60 $\mathrm{mm} \mathrm{Hg}$, temperatura corporal $36.4^{\circ} \mathrm{C}$, frecuencia del pulso 88 latidos por minuto, frecuencia respiratoria, 18 excursiones por minuto $\mathrm{y}$ saturación de oxígeno 98\% ( $\mathrm{FiO}_{2}$ ambiental). Cuello: central, no ingurgitación yugular, no masas ni ganglios. Piel: ligeramente fría, híperpigmentación cutánea y de las mucosas de los labios y encías (Figuras 1a y 1b). Lesión pustulosa a nivel del escroto izquierdo, con orificio de salida de $10 \mathrm{~mm}$ de diámetro y drenaje de material purulento. Resto del examen sin alteraciones significativas.

Exámenes auxiliares: hemoglobina, hemograma y las pruebas de función renal y hepática fueron normales. El análisis de orina mostró solo leucocituria. Los niveles de cortisol y hormona adrenocorticótropa estuvieron alterados más hiponatremia. Ver tabla 1.

La ultrasonografía testicular mostró al epidídimo izquierdo marcadamente heterogéneo, incrementado de tamaño, gran

Tabla I. Exámenes de laboratorio.

\begin{tabular}{|c|c|c|}
\hline & Valor & Rango referencial \\
\hline Hematocrito (\%) & 44,5 & $40-50$ \\
\hline Leucocitos $\left(10^{3}\right.$ por $\left.\mathrm{mm}^{3}\right)$ & 5500 & $5,0-10$ \\
\hline Plaquetas $\left(10^{3}\right.$ por $\left.\mathrm{mm}^{3}\right)$ & 275 & $150-450$ \\
\hline Glucosa (mg/dL) & 93,2 & $70-100$ \\
\hline Creatinina (mg/dL) & 0,8 & $0,6-I, 2$ \\
\hline Urea (mg/dL) & 22,8 & $16,6-48,5$ \\
\hline Albúmina (g/dL) & 4,72 & $3,5-5,2$ \\
\hline TGO / TGP (U/L) & $19,5 / 32$ & $<40$ \\
\hline GGTP (U/L) & 22 & $<60$ \\
\hline Fosfatasa alcalina (U/L) & 70,3 & $40-130$ \\
\hline Tiempo de coagulación (min) & 7 & $5-8$ \\
\hline Tiempo de sangría (min) & 2 & $1-3$ \\
\hline Sodio sérico $\mathrm{mEq} / \mathrm{L}$ & 132 & $135-148$ \\
\hline Potasio $\mathrm{mEq} / \mathrm{L}$ & 4,7 & $3,5-5,3$ \\
\hline Cloro mEq/L & 97 & $96-111$ \\
\hline Cortisol sérico am (ug/dL) & 4,59 & $6,2-19,4$ \\
\hline ACTH $(\mathrm{pg} / \mathrm{mL})$ & 1980 & $7,2-63,3$ \\
\hline TSH ultrasensible (pg/mL) & 4,21 & $0,27-4,20$ \\
\hline Tiroxina libre (ng/dL) & 1,32 & $0,93-1,70$ \\
\hline VIH Antigeno Anticuerpo (COI) & 0,233 & $0-0,9$ \\
\hline
\end{tabular}
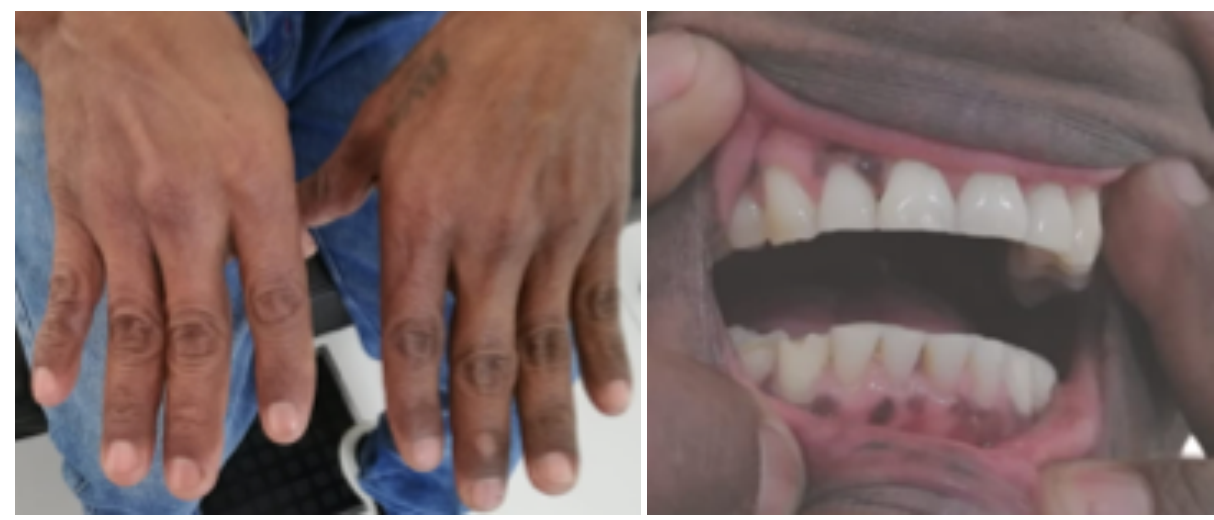

Figura I. Hiperpigmentación cutánea y de las mucosas de los labios y encías 
vascularidad, el cuerpo medía 19 × $19 \mathrm{~mm}$, presentaba calcificaciones en su interior y con trayecto fistuloso a la pared escrotal. La radiografía de tórax no mostró hallazgos patológicos (Figura 2). La tomografía abdominal (figuras 3 a y 3 b) reveló a la glándula suprarrenal derecha agrandada, de 34,9 x 20,6 mm (107 hu), con índices de atenuación en relación a componente sólido y captación irregular del medio de contraste; y, la glándula suprarrenal izquierda engrosada con dos pequeños nódulos sólidos, captadores en forma heterogénea de contraste. El resto de órganos fueron normales.

Bajo el diagnóstico orquiepididimitis y fístula escrotal se hizo interconsulta al servicio de urología, quien

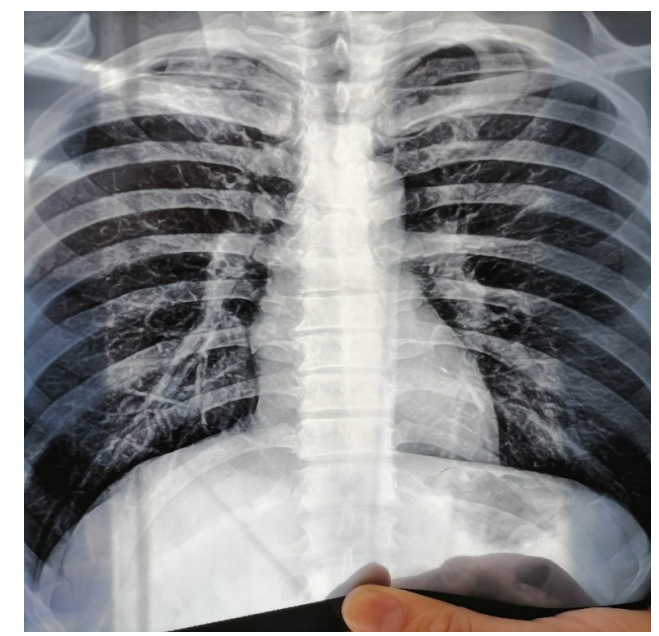

Figura 2. Radiografía de tórax: sin alteraciones significativas. planteó orquiectomía radical izquierda. El estudio anatomopatológico reveló, con la tinción de hematoxilina eosina, enfermedad crónica granulomatosa tuberculoide con células gigantes multinucleadas de tipo Langhans y con necrosis de aspecto caseoso que comprometía el parénquima hasta la albugínea (Figuras 4a y 4b).

Ante un paciente con síntomas generales, híperpigmentación cutánea y de mucosas, disminución de peso, síntomas gastrointestinales, astenia e hiponatremia, como el nuestro, era necesario descartar la insuficiencia suprarrenal primaria. Nuestra sospecha fue respaldada por los niveles de cortisol y ACTH en sangre previo al inicio de tratamiento.

Su tratamiento inicial requirió reposición hidroelectrolítica y corticoterapia de sustitución, y el tratamiento de la tuberculosis con esquema cuádruple según el protocolo del Programa de Control de la Tuberculosis. ${ }^{5}$ El paciente evolucionó favorablemente y fue dado de alta completamente recuperado.

\section{DISCUSIÓN}

La tuberculosis es la enfermedad infecciosa más extendida y una de las principales causas de mortalidad de enfermedades infecciosas en todo el mundo. Durante los últimos años, se ha venido informando un aumento de la enfermedad extrapulmonar. ${ }^{6}$ Rara vez se ha informado de tuberculosis sistémica que causa agrandamiento e insuficiencia suprarrenal. Las principales manifestaciones de la insuficiencia suprarrenal son la hiperpigmentación en el pliegue palmar, nudillos y mucosa bucal, trastornos gastrointestinales asociadas a hiponatremia e
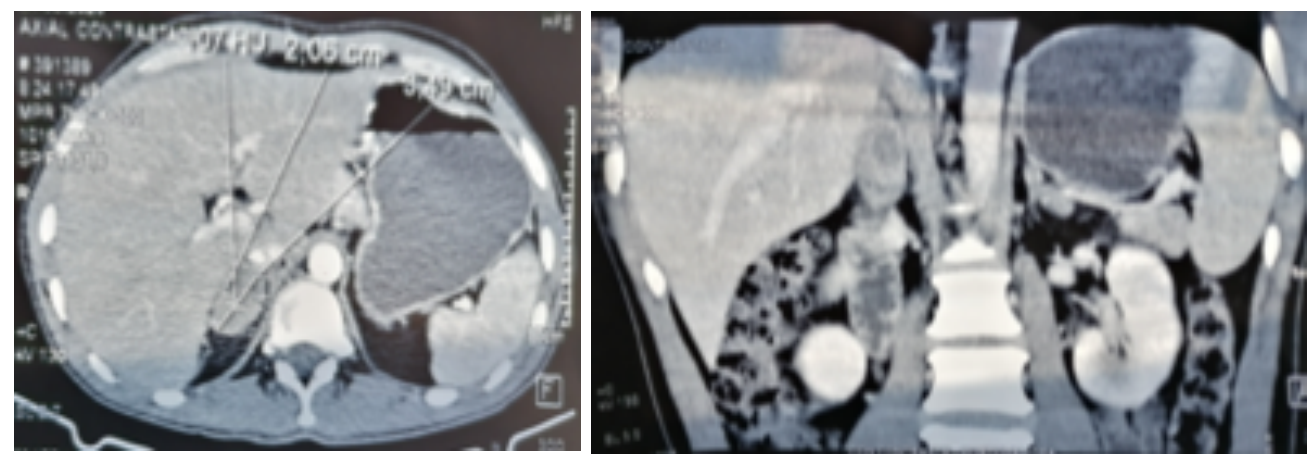

Figuras $\mathbf{3 a}$ y $\mathbf{3 b}$. TEM abdominal con contraste: glándula suprarrenal derecha agrandada.
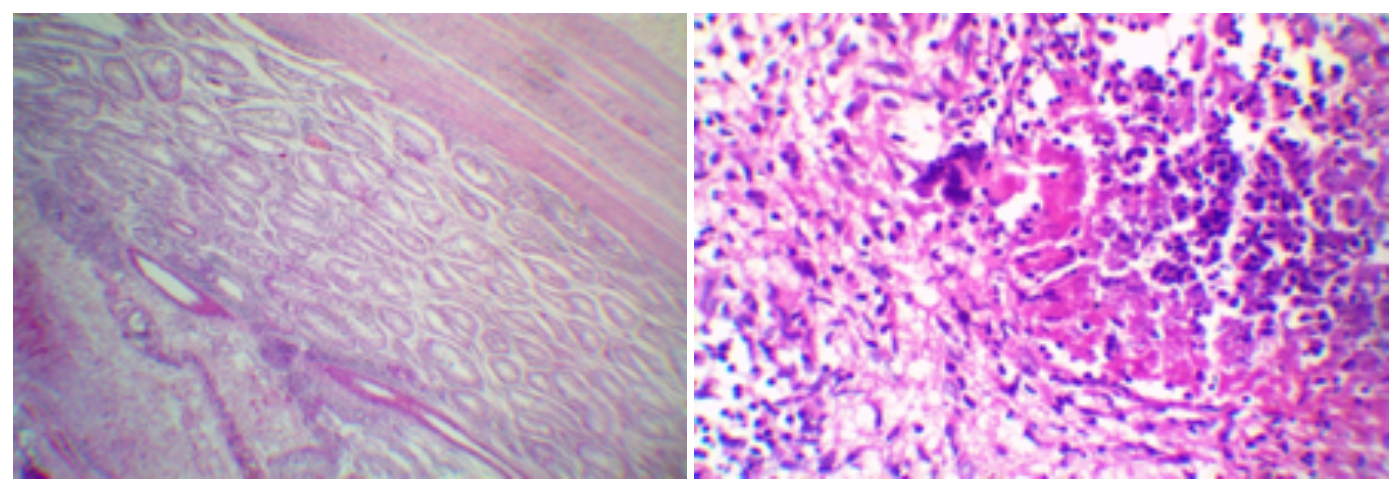

Figuras 4a y 4b. Tejido de testículo izquierdo. Tinción H-E, aumentos 40x y 400x: áreas de lesión granulomatosa tuberculoide, células gigantes y necrosis. 
hipercalcemia. $^{7}$

$\mathrm{Si}$ bien a nivel mundial se describe a la adrenalitis autoinmune como la causa más frecuente de insuficiencia suprarrenal, en países en vías de desarrollo como el nuestro la tuberculosis es la causa más frecuente. ${ }^{8,9}$ La tomografía computarizada es la técnica diagnóstica importante para el estudio de patología suprarrenal por su alta resolución. El diagnóstico de tuberculosis adrenal usando la tomografía en asociación con otros análisis de laboratorio y confirmada con el resultado de la biopsia ha sido descrita. ${ }^{9}$ En nuestro caso no se pudo confirmar la presencia de tuberculosis primaria pulmonar ni adrenal, siendo la tomografía el examen que hizo sospechar nuestro diagnóstico y, después, fue confirmado con la biopsia testicular. No se realizó biopsia suprarrenal porque las manifestaciones eran sugerentes de tuberculosis suprarrenal con tuberculosis extraadrenal activa.

El mecanismo de diseminación del bacilo tuberculoso hacia las estructuras del saco escrotal es controvertido. Se cree que, en la mayoría de los casos, la orquieepididimitis tuberculosa se desarrolla a partir de la diseminación retrógrada del bacilo tuberculoso desde el tracto urinario afectado hacia la próstata a través del reflujo, seguida de diseminación canalicular a la vesícula seminal, el conducto deferente y el epidídimo. Sin embargo, los bacilos de la tuberculosis también pueden ingresar a través de la diseminación hematógena y linfática. ${ }^{4}$

La tuberculosis con compromiso suprarrenal es el resultado de una diseminación hematógena temprana $\mathrm{y}$, a menudo, se asocia con una infección extraadrenal. ${ }^{10}$ Así, en un reporte de una serie autopsias, el $6 \%$ de los pacientes con tuberculosis activa tenía evidencia de infección suprarrenal; además, se hizo hincapié que cuando la infección no se circunscribe a las suprarrenales, las manifestaciones extraadrenales más frecuentes son la tuberculosis pulmonar y genitourinaria. ${ }^{11}$ Nuestro paciente solo presentó cuadro clínico de compromiso adrenal y genitourinario.

Las recomendaciones de tratamiento para la tuberculosis suprarrenal con cepas sensibles a fármacos son similares a las descritas para la tuberculosis pulmonar en todos los casos. La rifampicina induce las enzimas hepáticas responsables del metabolismo de los esteroides, lo que aumenta potencialmente el metabolismo de los glucocorticoides. ${ }^{12,13}$

La tuberculosis sigue siendo una de las principales causas de insuficiencia suprarrenal. La rápida intervención, un alto índice de sospecha con la filiación y el tratamiento precoz de la tuberculosis pueden permitir la recuperación de la función suprarrenal. En conclusión, la tuberculosis sistémica que resulta en insuficiencia suprarrenal debe considerarse en el escenario clínico adecuado.

\section{REFERENCIAS BIBLIOGRÁFICAS}

I. Coras Natalia, Somocurcio José, Aguilar Cristian. Tuberculosis primaria de la glándula suprarrenal:comunicación de dos casos.An Fac med. 2013; 74(3): 22I-226. URL disponible en: http:// www.scielo.org.pe/scielo.php?script=sci_arttext\&pid=SI025558320 I 30003000 I I \&lng=es.
2. Halperin I, Ortega E. Enfermedades de las glándulas suprarrenales. Medicina Interna. I7 $7^{\text {. }}$ ed. Elsevier; p. 1912-6.

3. Cerda-Guerrero E.J., Rodríguez-Rivera J.A. Tuberculosis testicular presentación de un caso y revisión de la literatura. Rev Mex Urol. 2020;80(3): I-9.

4. Das A, Batabyal S, Bhattacharjee S, Sengupta A.A rare case of isolated testicular tuberculosis and review of literature.J Family Med Prim Care. 2016;5(2):468-70. doi: https://dx.doi. org/I0.4I03\%2F2249-4863. 192334

5. Norma técnica de salud para la atención integral de las personas afectadas por tuberculosis. Ministerio de Salud. Dirección General de Salud de las Personas. Estrategia Sanitaria Nacional de Prevención y Control de la Tuberculosis, Lima: Ministerio de Salud; 2018.

6. Reid MJA, Arinaminpathy N, Bloom A, et al. Building a tuberculosisfree world: The Lancet Commission on Tuberculosis. Lancet. 2019; 393, I0178, I331-84.

7. Al-Mamari A, Balkhair A, Gujjar A, Ben Abid F, Al-Farqani A, AlHamadani A, Jain R.A case of disseminated tuberculosis with adrenal insufficiency. Sultan Qaboos Univ Med J. 2009 Dec;9(3):324-7. Epub 2009 Dec 19. PMID: 2 I 5093 I8; PMCID: PMC3074785.

8. Noe Israel Atamari Anahui, Manuel Montoya Lizárraga. Insuficiencia suprarrenal primaria (enfermedad de Addison) secundaria a tuberculosis suprarrenal. Revista del Cuerpo Médico Hospital Nacional Almanzor Aguinaga Asenjo. 20 I 4; 7(I):33-35.

9. Tinoco-Solórzano A, Córdova- Valenzuela V, Carrillo-Sanabria C, Meza-Legua F, Dávila-Aranda B. Diagnostico tomográfico de tuberculosis adrenal primaria en enfermedad de Addison en la altitud. Reporte de caso. Rev Fac Med Hum. 2020;20(4):727-730. doi: 10.25176/rfmh.v20i4.2953

10. Ramírez-Lapausa M., Menéndez-Saldaña A., NogueradoAsensio A. Tuberculosis extrapulmonar, una revisión. Rev esp sanid penit. 20I 5; I7(I):3-I I. URL disponible en: http://scielo.isciii.es/scielo. php? script=sci arttext\&pid=SI 575-62020 I 5000 I 00002\&Ing=es innard C, Blumberg EA. Endocrine and Metabolic Aspects of Tuberculosis. Microbiol Spectr. 2017 Jan;5(I): I0.II28/microbiolspec. TNMI7-0035-20 I6. doi: I0. I I28/microbiolspec.TNMI7-0035-20 I6. PMID: 282335I0; PMCID: PMC5785I 04.

II. Upadhyay J, Sudhindra P, Abraham G, Trivedi N. Tuberculosis of the adrenal gland: a case report and review of the literature of infections of the adrenal gland. Int J Endocrinol. 20|4;20 I4:876037. doi: 10.II55/20I4/876037. Epub 2014 Aug 6. PMID: 25I65474; PMCID: PMC4I 38934.

12. Nahid P, Dorman SE, Alipanah N, Barry PM, Brozek JL, Cattamanchi A, et al. Official American Thoracic Society/Centers for Disease Control and Prevention/Infectious Diseases Society of America Clinical Practice Guidelines: Treatment of Drug-Susceptible Tuberculosis. Clin Infect Dis. 2016 Oct I;63(7):el47-el95. doi: 10.1093/cid/ciw376. Epub 2016 Aug I0. PMID: 275I6382; PMCID: PMC6590850.

\section{CORRESPONDENCIA:}

Halbert Christian Sánchez Carrillo

hsc2005@hotmail.com

Fecha de recepción: 28-08-202I.

Fecha de aceptación: 02-II-202I.

Fuentes de financiamiento: por los autores.

Conflictos de interés: ninguno, según declaración de los autores.

Aspectos éticos: se obtuvo el consentimiento informado del paciente y de sus familiares.

Contribuciones de los autores: los tres autores contribuyeron por igual en la concepción, redacción, revisión y aprobación final del manuscrito. 